POS PROCEEDINGS

\title{
Radio emission in ultracool dwarfs
}

\author{
J. B. Climent* \\ Departament d'Astronomia i Astrofísica, Universitat de València, C. Dr. Moliner 50, 46100 \\ Burjassot, València, Spain \\ E-mail: j.bautista.climent@uv.es
}

\section{J. C. Guirado, R. Azulay, B. Gauza, M. A. Pérez-Torres, R. Rebolo, M. R. Zapatero Osorio, J. M. Marcaide and I. Martí-Vidal}

\begin{abstract}
Previous observations of Ultracool Dwarfs at radio wavelengths opened a new window to probe the nature of their radio emission. Despite the fact that VLBI observations are still scarce in this field, they provide unique insights on the mechanisms responsible for the detected emission and, at the same time, they allow a better characterization of the orbital motion in binary or triple systems as well as their dynamical masses. In this EVN Symposium we present VLA + EVN observations of the triple system VHS 1256-1257 and LBA + AMBER/VLTI observations of the binary system AB Dor A/C.

We discovered radio emission in VHS 1256-1257 at X-band spatially coincident with the central binary brown dwarf and determined the spectral index which suggests that non-thermal, opticallythin, synchrotron or gyrosynchrotron radiation was the responsible mechanism. In addition, we constrained the magnetic field intensity to be of the order of a few $\mathrm{kG}$.

Regarding $\mathrm{AB}$ Dor $\mathrm{A} / \mathrm{C}$, the VLBI data clearly showed a substructure in the main star of this binary system (AB Dor A). AMBER data showed evidence for AB Dor $\mathrm{C}$ being not a point source but actually a binary system itself.
\end{abstract}

14th European VLBI Network Symposium \& Users Meeting (EVN 2018)

8-11 October 2018

Granada, Spain

${ }^{*}$ Speaker. 


\section{Introduction}

The discovery of the first radio emission from an ultra-cool dwarf (UCD) proved the existence of powerful magnetic fields ( $\sim \mathrm{kG})$ on these objects (Berger et al. 2001). Subsequent radio emission studies of ultracool objects (late M, L, and T objects; e.g. Matthews 2013) opened a new window to understand their magnetism both phenomenologically (quiescent emission, coherent bursts...) and physically (auroral, gyrosynchrotron and planet-like radio emission; see Williams 2017 and references therein). This knowledge is crucial for planet discovery around them (Robertson et al. 2014) and for the atmosphere and habitability of these planets (Jakosky et al. 2015; Shields et al. 2016) which could be abundant and observationally accessible (e.g. TRAPPIST-1 system, see Gillon et al. 2016, 2017). Moreover, the study of UCD may open a suitable route to the detection of direct exoplanet radio emission: while no exoplanet has been yet detected at radio wavelengths, an increasing number of UCD (Hallinan et al. 2008; Pineda et al. 2017; Guirado et al. 2018) show substantial evidence of radio emission at $\mathrm{GHz}$ frequencies in objects with spectral types as cool as T6.5 (Kao et al. 2016), including a likely planetary-mass T2.5 object (Kao et al. 2018).

VHS J125601.92-125723.9 (hereafter VHS 1256-1257; Gauza et al. 2015) is a triple system composed by a $0.1^{\prime \prime}$ equal-magnitude M7.5 brown dwarf binary (components $\mathrm{A}$ and B) and a lowermass L7 companion (component b) separated $8^{\prime \prime}$ from the primary pair. The system is relatively young, 150-300 Myr, and nearby (12.7-17.1 pc), which locates the low-mass object b near the deuterium burning limit (Stone et al. 2016). The new trigonometric distance of $15.8 \mathrm{pc}$, obtained using optical and near-infrared images spanning a few years, is the one we will adopt (Zapatero Osorio et al. 2017). This distance is compatible with a likely age of $300 \mathrm{Myr}$, which agrees with the strong lithium depletion observed in the high resolution spectra of the primary double brown dwarf and the recent age determinations of Stone et al. (2016) and Rich et al. (2016). The luminosity of the very low mass companion VHS 1256-1257 b can be estimated making use of the bolometric corrections available for red L dwarfs (Filippazzo et al. 2015) and the new distance adopted which yields a value of $\log L / L_{\odot}=-5.02 \pm 0.10$. According to the luminosity-mass-age relationship of Chabrier et al. (2000), for an age range of 150-300 Myr, this luminosity value implies a mass in the interval of 10-20 $M_{J}$, with a most likely value of $12-15 M_{J}$ at $300 \mathrm{Myr}$.

It is important to note that VHS 1256-1257 holds a special interest due to various factors: i) it is only the third multiple system known with all components being substellar objects (Bouy et al. 2005; Radigan et al. 2013); ii) the population of L7 objects is an intriguing one with high content of atmospheric dust or high metallicity (Rich et al. 2016); iii) due to the large separation between the main central pair and the L7 object, they can be distinguishable at virtually all wavelengths; iv) as mentioned above, the binarity of the central pair will allow us a good determination of their dynamical masses in a few years, which is essential to fully characterize the system. For these reasons, we submitted and analyzed Karl G. Jansky Very Large Array (VLA) and European VLBI Network (EVN) observations of VHS1256-1257.

About 15 pc away, AB Doradus is a pre-main-sequence (PMS) system formed by two pairs of stars separated by 9", AB Dor A/C and AB Dor Ba/Bb (Close et al. 2005; Guirado et al. 2006), giving name to the $\mathrm{AB}$ Doradus moving group ( $\mathrm{AB}$ Dor-MG). The main star of this system, the $\mathrm{K} 0$ dwarf AB Dor A is a weak-line T Tauri star (Herbst \& Shevchenko) with a rotation period of $\sim 0.5$ 
days (Hussain et al. 2007; Plavchan et al. 2009) and presents strong emission at all wavelengths, from radio to X-rays. It has been well studied by the Hipparcos satellite (Lestrade et al. 1995) and very-long-baseline-interferometry (VLBI) arrays (Lestrade et al. 1995; Guirado et al. 1997). This joined effort revealed the presence of $\mathrm{AB}$ Dor $\mathrm{C}$, a low-mass companion with $0.090 M_{\odot}$, orbiting $\mathrm{AB}$ Dor $\mathrm{A}$ at an average angular distance of $0.2^{\prime \prime}$. The pair AB Dor A/C was also observed by different near-infrared instruments at the VLT (Close et al. 2005; Close et al. 2007; Boccaletti et al. 2008) allowing independent photometry of $A B$ Dor $C$ which, along with the dynamical mass determination, served as a benchmark for stellar evolutionary models. The detection (or lack of it) of the UCD AB Dor $\mathrm{C}$ at radio wavelenghts would inform us about the possible mechanisms that create this type of emission and may allow us to estimate the magnetic field in this object. Another motivation for the study of the binary system $\mathrm{AB}$ Dor $\mathrm{A} / \mathrm{C}$ is the possible binarity of $\mathrm{AB}$ Dor $\mathrm{C}$ which, along its uncertain age, complicates an accurate calibration of the evolutionary models. These models tend to overestimate the luminosity (or underestimate the mass) of young, low-mass objects. In the case of AB Dor C, this discrepancy could be easily corrected if this object were indeed a binary system with a mass ratio of $\sim 3$ (Marois et al. 2005). Given the crucial part that these objects play in calibration of the models, this binary hypothesis will be studied in detail with the AMBER/VLTI data.

Please, note that the results of the triple system VHS 1256-1257 have been published in Guirado et al. (2018) and the results of the binary AB Dor A/C will be published in more detail soon (Climent et al. in prep).

\section{Observations and data analysis}

\subsection{VHS 1256-1257 observations}

The system VHS 1256-1257 was observed with the VLA at X- and L-band on 2015 May 15 and 2016 Jul 28, respectively. The observation at X-band lasted 2 hours and was carried out in BnA configuration, using an effective bandwidth of $4 \mathrm{GHz}(8-12 \mathrm{GHz})$ in dual polarization. The observation at L-band lasted 1 hour, in B configuration, and using an effective bandwidth of $1 \mathrm{GHz}$ $(1-2 \mathrm{GHz})$ in dual polarization. The data were reduced using the CASA software package of the NRAO following the standard procedure of calibration for continuum VLA data.

After the detection of radio emission from the VLA data we performed multiepoch (4 Mar 2016, 27 May 2016 and 2 Nov 2016) observations with the EVN at L-band. Each observation lasted for 6-7 hr with dual polarization and phase-reference mode. The data were reduced using the program Astronomical Image Processing System (AIPS) of the National Radio Astronomy Observatory (NRAO) with standard routines. Once the final data were calibrated, the images were made with the Caltech imaging program DIFMAP (Shepherd et al. 1994).

\subsection{AB Dor A/C observations}

We observed the binary system AB Dor A/C using the Australian Long Baseline Array (LBA) in dual polarization at $1.4 \mathrm{GHz}$ during February 6th, 2018 for a total duration of 10.5 hours in phase-reference mode. Five antennas were used (At, Ho, Cd, Mp and Pa) although Mp did not observe since the L-band receiver was not available. We reduced and analyzed the data using AIPS with standard routines. The final images were made with DIFMAP. 
The observations of AB Dor C were performed with the VLT-Interferometer (VLTI) using the AMBER instrument (Astronomical Multi-BEam combineR; Petrov et al. 2007) with the external fringe tracker FINITO (Fringe-Tracking Instrument of NIce and TOrino). We employed the low resolution mode in the J, $\mathrm{H}$, and $\mathrm{K}$ bands. We observed on the 27 December 2012 using the $8.2 \mathrm{~m}$ unit telescopes (UT) with the configuration UT1-UT2-UT4. Due to the faint magnitude of AB Dor $\mathrm{C}$, we had to use a non-standard configuration with $\mathrm{AB}$ Dor $\mathrm{A}$ as a fringe tracker to increase the integration time on $\mathrm{AB}$ Dor $\mathrm{C}$. The data were reduced using the software package amdlib v.3.0.8 (Tatulli et al. 2007; Chelli et al. 2009). The details of this reduction will be given in Climent et al. (in prep).

\section{Results and discussion}

\subsection{VHS 1256-1257}

We detected the central equal mass brown dwarf binary on 2015 May 15 (X-band) as an unresolved source with a peak flux density of $60 \mu \mathrm{Jy}$ (Figure 1). We made use of the coordinates and proper motion given in Gauza et al. (2015) to find the expected position of component $A B$ at the time of our observation. We did not detect the L7 companion with a noise floor of $\sim 3 \mu \mathrm{Jy}$ at the expected position which imposes a strong upper bound to its radio emission. We found no detection in any of the components of the system at L-band neither with the VLA nor with EVN, placing a $3 \sigma$ threshold detection of $20 \mu \mathrm{Jy}$. The flux density measured at X-band implies a radio luminosity of $1.95 \cdot 10^{-13} \mathrm{ergs}^{-1} \mathrm{~Hz}^{-1}$ at $15.8 \mathrm{pc}$. If this flux is originated at only one the components, this luminosity is similar to other single UCDs detected with comparable spectral types (M7.5; McLean et al. 2011).

Under the assumption that radio emission comes from a single brown dwarf of size $0.12 R_{\odot}$ (derived from the models of Chabrier et al. 2000), we calculate a brightness temperature of $5.4 \cdot 10^{7} \mathrm{~K}$ (x2 for equal binary contribution), which is consistent with synchrotron or gyrosynchrotron nonthermal radio emission (Dulk 1985). The low degree of circular polarization (less than 15\%) seems to discard coherent mechanisms predicted for ultracool dwarfs (i.e., auroral emissions; Hallinan et al. 2015; Kao et al. 2016).

In addition, in the X-band observations we produced four narrower-band images of VHS 12561257 by deconvolving adjacent $1 \mathrm{GHz}$-bandwidth data sets separately from which we obtained an estimation of the spectral behaviour of this system between 8 and $12 \mathrm{GHz}$ (Figure 2). The corresponding spectral index is $\alpha=-1.1 \pm 0.3\left(S \propto v^{\alpha}\right)$, compatible with optically thin non-thermal synchrotron or gyrosynchrotron emission from a power-law energy distribution of electrons. The lack of detection at L-band led us to formulate a hypothesis consisting in considering that the radio emission is actually self-absorbed at this frequency. Using the analytic expressions developed by White et al. (1989) for gyrosynchrotron radio emission of dMe stars in quiescent conditions, along with our estimation for the spectral index, we found two values for the spectral index of the optically thick component of the radiation, $\alpha=0.6$, and $\alpha=1.2$, which imply magnetic field intensities in the range of 1.2-2.2 kG. This range agrees with brown-dwarf magnetic field estimates derived from theoretical models (Reiners et al.2010a) and with the average value of the magnetic field intensity found in a sample of M7-9.5 dwarfs (Reiners et al. 2010b). 

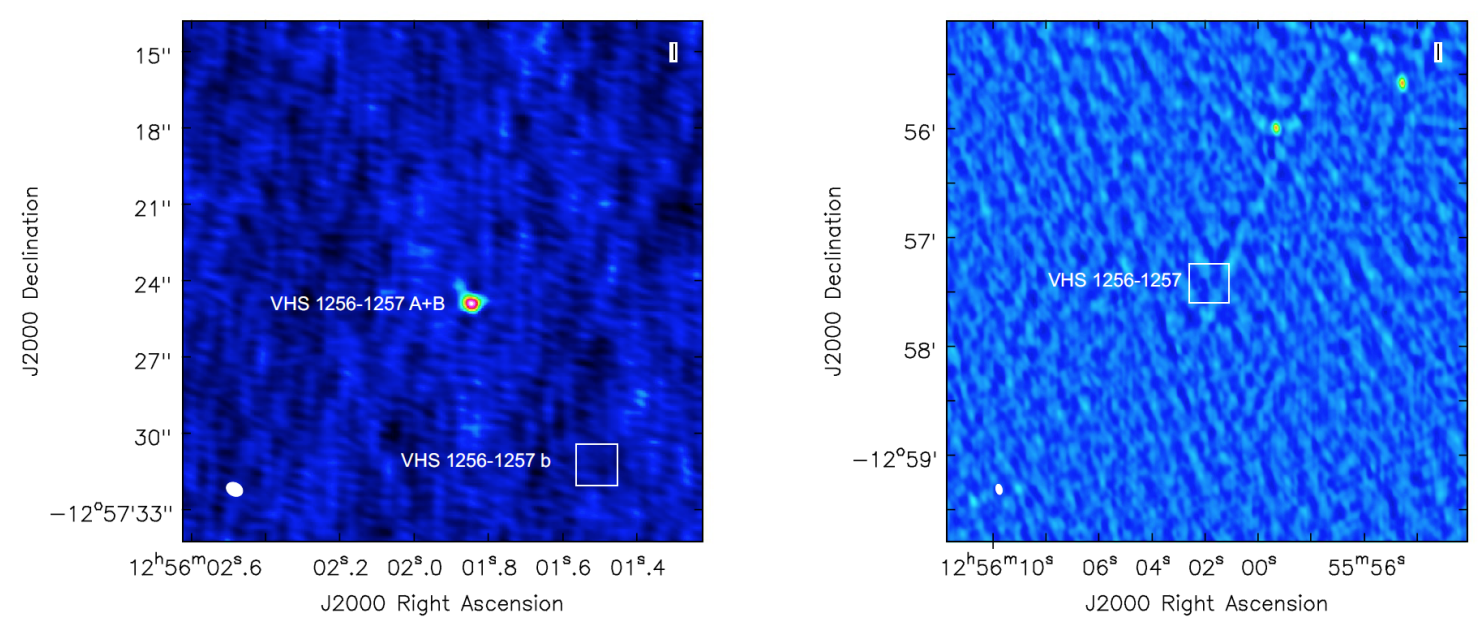

Figure 1: Left. VLA image of the VHS1256-1257 field at X-band. The detected source corresponds with the brown dwarf binary VHS1256-1257 AB. The location of the (undetected) L7-object $b$ is marked with a solid white box. The $3 \sigma$ threshold detection is $9 \mu \mathrm{Jy}$. Right. VLA image of the VHS1256-1257 field at L-band. A solid box, with size that of the X-band image, is centered at the position of the X-band detection. No detection is visible for any of the components with a $3 \sigma$ threshold detection of $20 \mu \mathrm{Jy}$

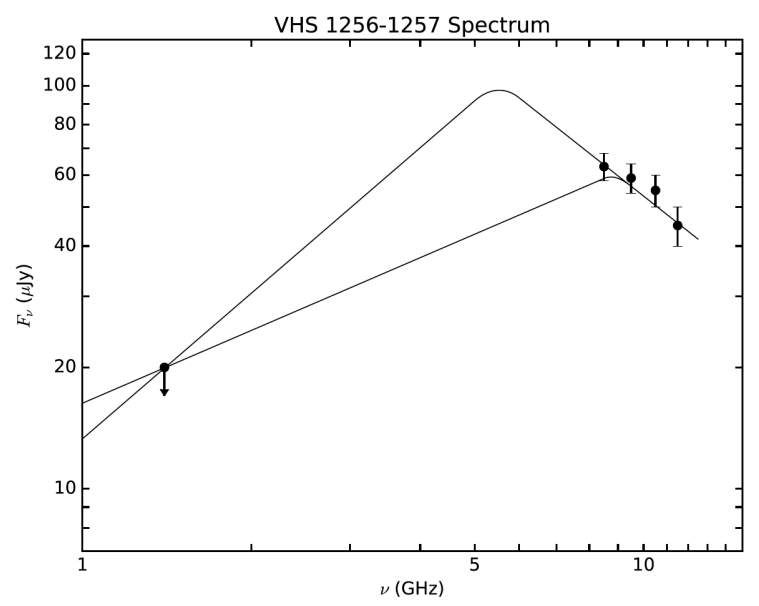

Figure 2: Spectrum of VHS 1256-1257 from VLA observations. The upper bound resulting from the nondetection at L-band is denoted with a downwards arrow. The solid lines illustrate two possible spectra, each one made from a combination of the behaviour predicted by the White et al. (1989) model of gyrosynchrotron emission (optically thin regime, showing the two extreme cases, $\alpha=0.6$ and 1.2; see text), and a fit to our flux density measurements (optically thick regime, $\alpha=-1.1$ ).

\subsection{AB Dor A/C}

The results of this binary system will be discuss in more detail in Climent et al. (in prep). Figure 3 clearly shows a substructure in the main component, AB Dor A, which may correspond to a dense coronal structure produced by the intense magnetic activity of this ultrafast rotating star. We found no emission at the expected position of $\mathrm{AB}$ Dor $\mathrm{C}$, according to the well-known orbit of the system (Guirado et al. 2006). Our non-detection at L-band places an upper limit of $60 \mu \mathrm{Jy}$ for 
the radio emission of this ultracool dwarf.

The values of the interferometric observables from AMBER data clearly show that AB Dor $\mathrm{C}$ is partially resolved on all 3 baselines (Figure 4). These oscilations are typical signatures of a binary system (e.g. Millour et al 2009). Therefore, we modelled the observed visibilities with a simple geometrical model of a binary system and fitted to the experimental data with an in-house software and, independently, with LITpro (Lyon Interferometric Tool prototype). Our preliminary results show that the best model is a binary system with 37 mas separation, P.A of 149.5 and a flux ratio of 0.05 .

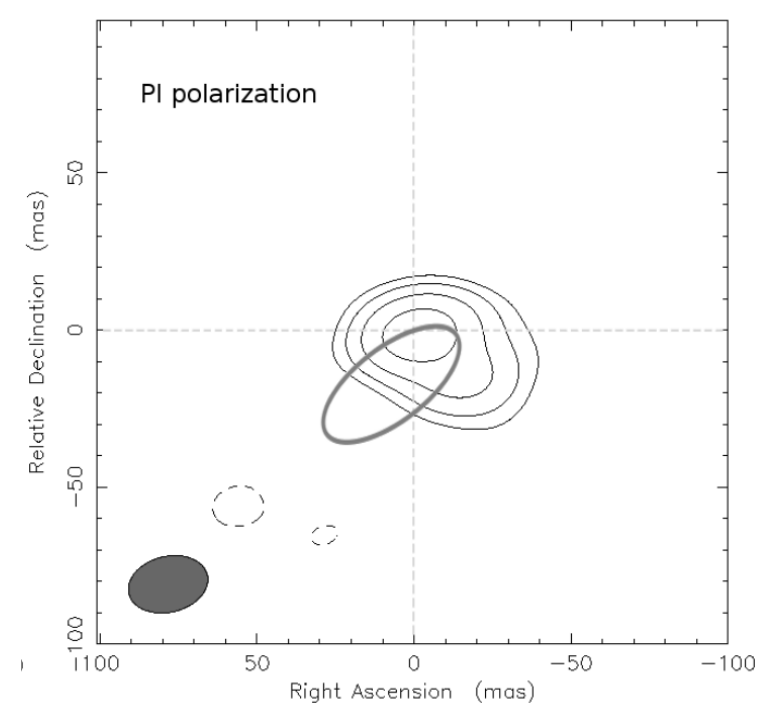

Figure 3: Reduced LBA $1.4 \mathrm{GHz}$ image of the binary system AB Dor A/C. The main star, AB Dor A, is centered in this image (R.A 5:28:44.976; DEC -65:26:52.398) and a substructure is visible on it in the southwest direction. We plot the absolute orbit of $\mathrm{AB}$ Dor $\mathrm{A}$ in grey, according to the best known values. The ultracool dwarf $\mathrm{AB}$ Dor $\mathrm{C}$ is not detected in any of the polarizations.

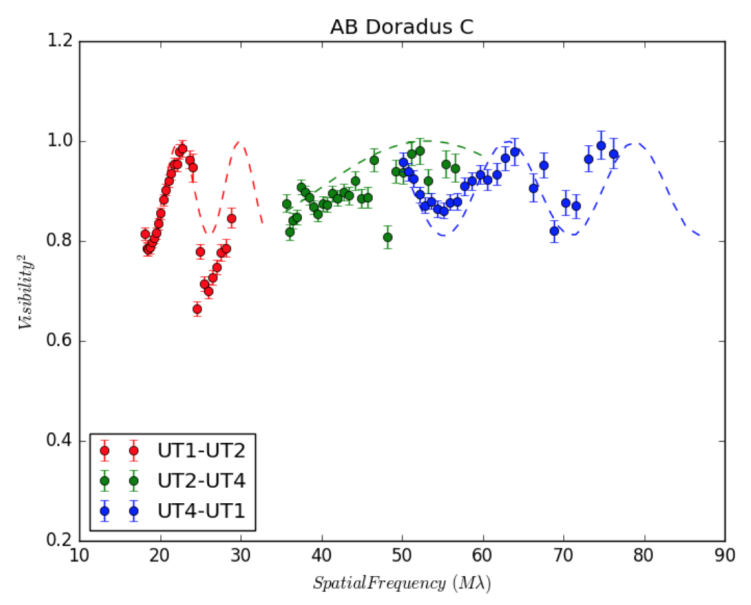

Figure 4: AMBER visibilities of AB Dor C. Different colors represent different baselines. The observational data (circles) are best fitted by a binary system (see text), represented by the discontinuous line. 


\section{Conclusions}

We have reported the detection of radio emission from the VHS1256-1257 system. The radio emission is originated at the central pair $\mathrm{AB}$, likely consisting in non-thermal gyrosynchrotron emission in presence of $\mathrm{kG}$ intense magnetic field. Regarding $\mathrm{AB}$ Dor $\mathrm{A} / \mathrm{C}$, our preliminary results reveal a substructure in the main component from LBA observations whose origin needs to be explained and our AMBER data show a strong hint for the binarity of the ultracool dwarf AB Dor C.

\section{References}

[1] Berger et al. 2001, Natur , 410, 338

[2] Boccaletti, A., Chauvin, G., Baudoz, P., \& Beuzit, J.-L. 2008, A\&A, 482, 939

[3] Bouy, H., Martín, E. L., Brandner, W., \& Bouvier, J. 2005, AJ, 129, 511

[4] Chabrier, G., Baraffe, I., Allard, F., \& Hauschildt, P. 2000, ApJ, 542, 464

[5] Close, L.M., Lenzen, R., Guirado, J.C. et al. 2005, Nature, 433, 286

[6] Filippazzo, J. C., Rice, E. L., Faherty, J., et al. 2015, ApJ, 810, 158

[7] Gauza, B., Béjar, V. J. S., Pérez-Garrido, A., et al. 2015, ApJ, 804, 96

[8] Gillon et al. 2016,Natur, 533, 221

[9] Guirado, J. C., Reynolds, J. E., Lestrade, J.-F., et al. 1997, ApJ, 490, 835

[10] Guirado, J. C., Martí-Vidal, I., Marcaide, J. M., et al. 2006, A\&A, 446, 733

[11] Guirado et al. 2018, A\&A, 610, A23

[12] Hallinan et al. 2008, ApJ, 684, 644

[13] Hussain G. A. J. et al., 2007, MNRAS, 377, 1488

[14] Jakosky et al. 2015, GeoRL, 42, 8791

[15] Kao et al. 2016, ApJ, 818, 24

[16] Kao et al. 2018, ApJS, 237, 25

[17] Lestrade, J.-F., Jones, D. L., Preston, R. A., et al. 1995, A\&A, 304, 182

[18] Matthews, L. D. 2013, PASP, 125, 313

[19] McLean, M., Berger, E., \& Reiners, A. 2012, ApJ, 746, 23

[20] Pineda et al. 2017, ApJ, 846, 75

[21] Plavchan, P., Werner, M. W., Chen, C. H., et al. 2009, ApJ, 698, 1068

[22] Rich, E. A., Currie, T., Wisniewski, J. P., et al. 2016, ApJ, 830, 114

[23] Radigan, J., Jayawardhana, R., Lafreniére, D., et al. 2013, ApJ, 778, 36

[24] Reiners, A., \& Christensen, U. R. 2010a, A\&A, 522, A13

[25] Reiners, A., \& Basri, G. 2010b, ApJ, 710, 924

[26] Robertson et al. 2014, Sci, 345, 440

[27] Shepherd, M. C., Pearson, T. J., \& Taylor, G. B. 1994, BAAS, 26, 987

[28] Shields et al 2016, Phys. Rep., 663, 1

[29] Stone, J. M., Skemer, A. J., Kratter, K. M., et al. 2016, ApJ, 818, L12

[30] Williams 2017 (arXiv:1707.04264) 